\title{
An Automatic Welding Defects Classifier System
}

\author{
Juan Zapata, Ramón Ruiz \\ Departamento de Electrónica y Tecnología de Computadoras. Universidad Politécnica de Cartagena, \\ Campus Muralla de Mar. Edificio Antiguo Cuartel de Antigones. Plaza del Hospital 1, 30202 Cartagena \\ Teléfono: 968326458, E-mail: juan.zapata@upct.es \\ Rafael Vilar \\ Departamento de Estructuras y Construcción. Universidad Politécnica de Cartagena, Campus Muralla del Mar. \\ Edificio Antiguo Hospital de Marina, Muralla del Mar s/n, 30202 Cartagena Teléfono: 968325636, E-mail_ \\ rafael.vilar@upct.es
}

\begin{abstract}
Radiographic inspection is a well-established testing method to detect weld defects. However, interpretation of radiographic films is a difficult task. The reliability of such interpretation and the expense of training suitable experts have allowed that the efforts being made towards automation in this field. In this paper, we describe an automatic detection system to recognise welding defects in radiographic images. In a first stage, image processing techniques, including noise reduction, contrast enhancement, thresholding and labelling were implemented to help in the recognition of weld regions and the detection of weld defects. In a second stage, a set of geometrical features was proposed and extracted between defect candidates. In a third stage, an artificial neural network for weld defect classification was used under three regularisation process with different architectures. For the input layer, the principal component analysis technique was used in order to reduce the number of feature variables; and, for the hidden layer, a different number of neurons was used in the aim to give better performance for defect classification in both cases.
\end{abstract}

\section{Experimental Method}

Figure 1 shows the major stages of our welding defect detection system. Digital image processing techniques are employed to lessen the noise effects and to improve the contrast, so that the principal objects in the image become more apparent than the background. Threshold selection methods, labelled techniques and feature extraction are used to obtain a feature discriminatory that can facilitate both the weld region and defects segmentation. Finally, fea tures obtained are input pattern to artificial neural network (ANN). Previously, principal component analysis (PCA) is first used to perform simultaneously a dimensional reduction and redundancy elimination. Secondly, an ANN is employed for the welding fault identification task where three regularisation processes are employed in order to obtain a better generalisation.

After digitising the films [1, 2], it is common practice to adopt a pre-processing stage for the images with the specific purpose of reducing/eliminating noise and improving contrast. Two pre-processing steps were carried out in this work: in the first step, for reducing/eliminating noise an adaptive Wiener filter [3] and Gaussian low-pass filter were applied, while for adjusting the image intensity values to a specified range to contrast stretch, contrast enhancement was applied in the second step.

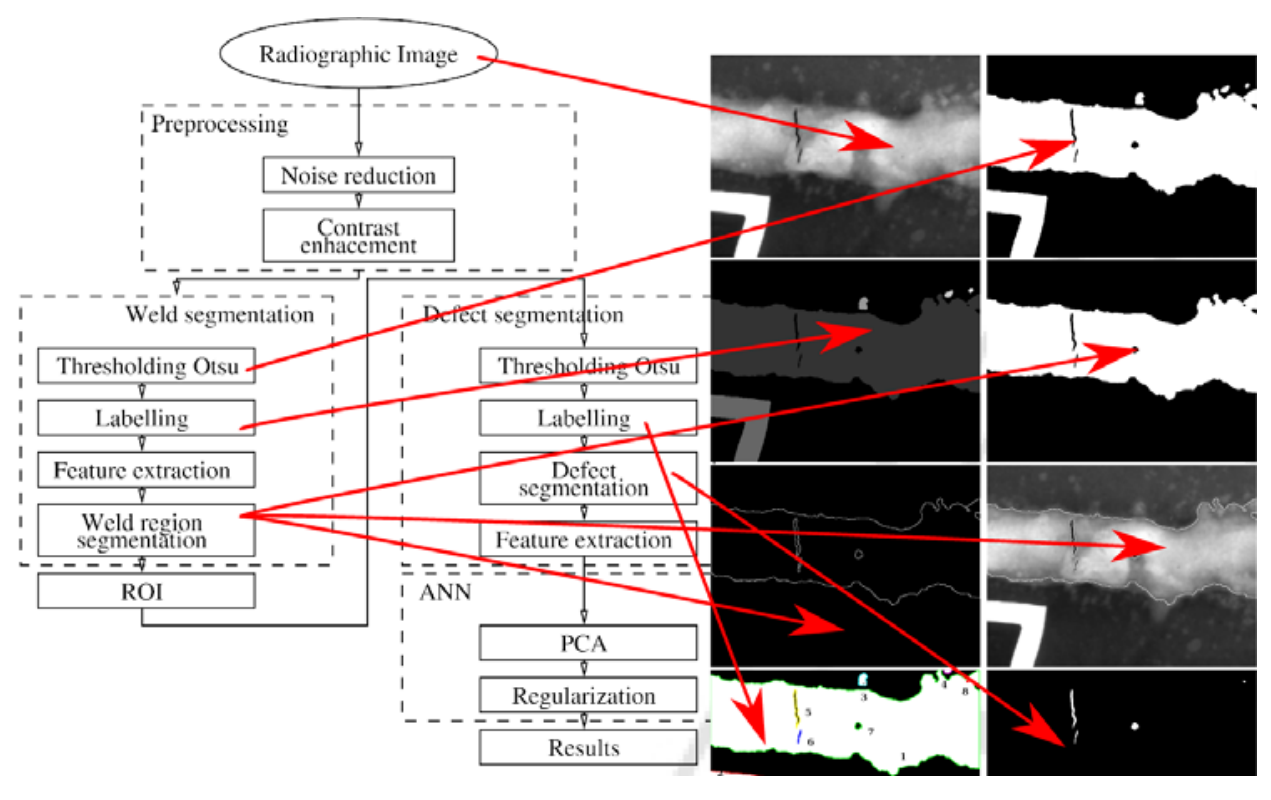


The last stage is the feature extraction in terms of individual and overall characteristics of the heterogeneities. The output of this stage is a description of each defect candidate in the image. This represents a great reduction in image information from the original input image and ensures that the subsequent classification of defect type and cataloguing of the degree of acceptance are efficient. In the present work, features describing the shape, size, location and intensity information of defect candidates were extracted.

The dimension of the input feature vector of defect candidates is large, but the components of the vectors can be highly correlated and redundant. It is useful in this situation to reduce the dimension of the input feature vectors. An effective procedure for performing this operation is principal component analysis. This technique has three effects: it orthogonalises the components of the input vectors (so that they are uncorrelated with each other), it orders the resulting orthogonal components (principal components) so that those with the largest variation come first, and it eliminates those components that contribute the least to the variation in the data set.

\section{Multi-layer Feed-forward ANN}

A multiply-input neuron model is shown on the left in Figure 2. The topology of the network used in this work is illustrated on the right in Figure 2. Nonlinear pattern classifiers were implemented using ANNs of the supervised type using the error backpropagation algorithm and two layers, one hidden layer ( $\mathrm{S}_{1}$ neurons) using hyperbolic tangent sigmoid transfer function and one output layer $\left(\mathrm{S}_{2}=\right.$ 5 neurons) using a linear transfer function. In this work, a BFGS algorithm [4] was used to train the network. The algorithm requires more computation in each iteration and more storage than the conjugate gradient methods, although it generally converges in fewer iterations. The approximate Hessian must be stored, and its dimension is $n \times n$, where $n$ is equal to the number of weights and biases in the network, therefore for smaller networks can be an efficient training function.

In this work, three methods were used in order to improve generalisation. The first method for improving generalisation is called regularisation with modified performance function. This involves modifying the performance function, which is normally chosen to be the sum of squares of the network errors on the training set. The second method automatically sets the regularisation parameters. It is desirable to determine the optimal regularisation parameters in an automated fashion. One approach to this process is the Bayesian regularisation $[5,6]$. The third method for improving generalisation is called early stopping or bootstrap. In this technique the available data is divided into three subsets. The first subset is the training set, which is used for computing the gradient and updating the network weights and biases, in our case $50 \%$ of data. The second subset is the validation set, $25 \%$ of data. The third subset, $25 \%$ of data, is the test set is not used during the training process.

\section{Result and Conclusions}

To validate the proposed technique for the automatic detection of weld defects, the same set of 86 radiograph images from the reference collection of the IIW/IIS were used. In order to evaluate the performance of the system, it is important to know if the system is able to detect all defects. In this stage, our system is able to obtain a sensibility of $100 \%$, i.e. the system detects as defect candidate all the defects observed by the human expert. For a defect detection system it is very important to have minimal loss in defect regions even at the cost of increasing the number of non-defect areas. The performance is obtained with a regression analysis between the network response and the corresponding targets. An artificial neural network can be more efficient if varying the number of
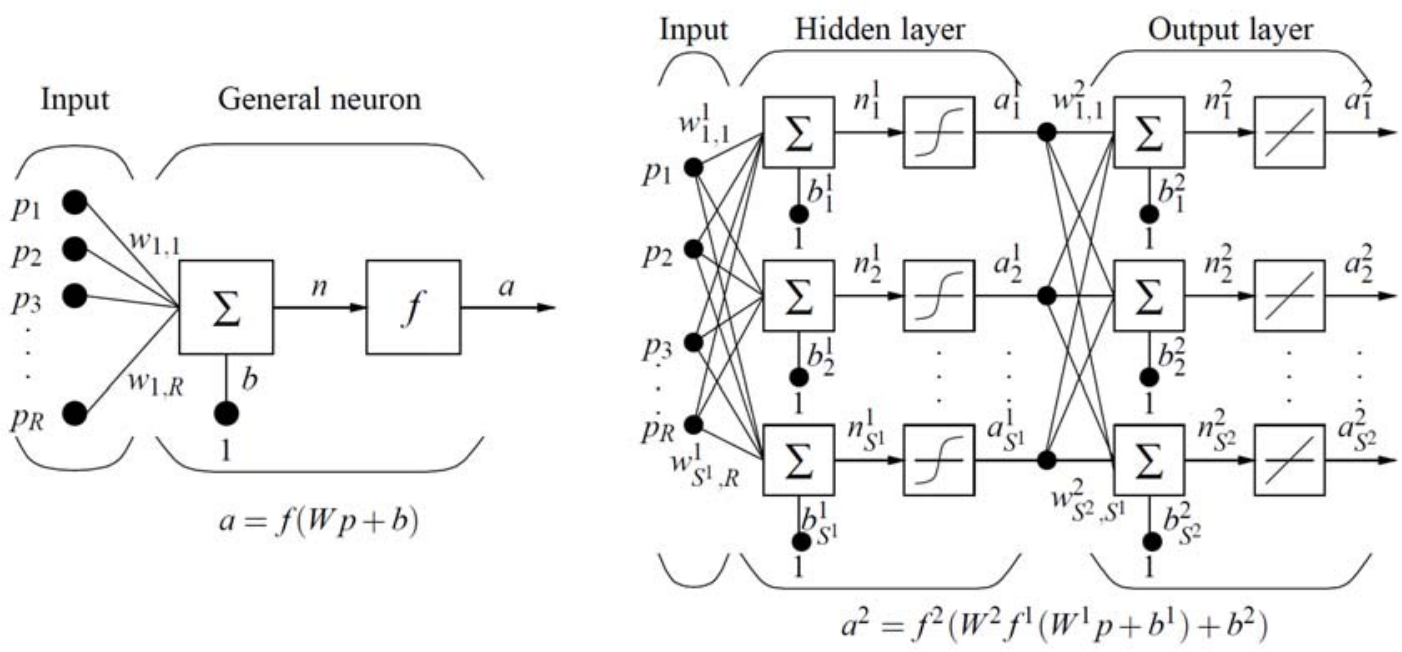

Figure 2: Neuron model and network architecture 

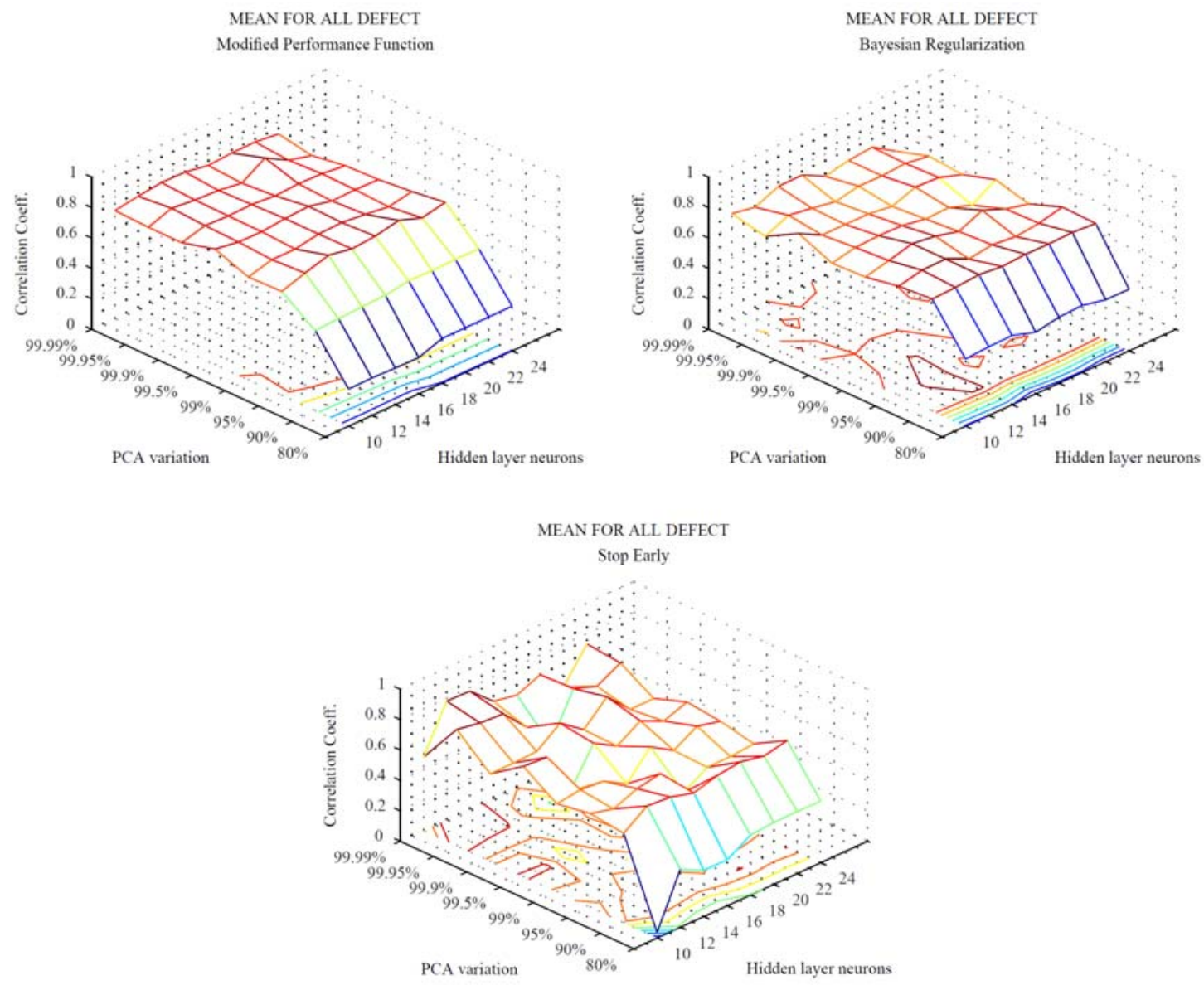

Figure 3: Mean Correlation coefficient for each regularisation method.

neurons $R$ in the input layer (by means of principal component analysis) and $S_{1}$ in the hidden layer and observing the performance of the classifier for each defect and for each method of regularisation was possible to obtain the most adequate number of neurons for the input and hidden layer and more appropriate method of regularisation.

Figure 3 illustrates the graphical output of correlation coefficient provided for the regression analysis for each method of regularisation, i.e. using a modified performance function, Bayesian regularisation and stopping early, in average for all classes. In general, all outputs seem to track the targets reasonably very well and all correlation coefficients are rounding 0.8 . Results indicate that the best implementation is a neural network with PCA with a conservation of $99.9 \%$ (11 input neurons) in the data information, a hidden layer with 20 neurons with a hyperbolic tangent sigmoid transfer function and an output layer with a linear transfer function. The regularisation method is based on a modified performance function with $\gamma=$ 0.5. The training algorithm is a quasi-Newton algorithm for fast optimisation based on the work of Broyden, Fletcher, Goldfarb and Shanno (BFGS algorithm).

\section{Referencias}

[1] Zscherpel, U. "Film digitisation systems for dir: Standards, requirements, archiving and printing”. 5 (05), $2000 \quad$ NDT.net (http://www.ndt.net).

[2] Zscherpel, U.. "A new computer based concep for digital radiographic reference images". 7 (12), 2002 NDT.net (http://www.ndt.net).

[3] Lim, J. Two-Dimensional Signal and Image Processing,. Prentice Hall, Englewood Cliffs. pages 536-540, 1990.

[4] Dennis, J. and Schnabel, R. Numerical Methods for Unconstrained Optimization and Nonlinear Equations. Prentice-Hall. 1983.

[5] MacKay, U, Bayesian interpolation. Neural Computation, 4(3); 415-447. 2002

[6] Foresee, F. and Hagan, M. "Gauss-Newton Approximation to Bayesian Regularization". In Proceedings of the 1997 International Joint Conference on Neural Networks, pages 1930 1935. 1997 